\title{
Aggregation Policies for Tuple Spaces
}

\author{
Kaminskas, Linas; Lluch Lafuente, Alberto
}

Published in:

COORDINATION 2018: Coordination Models and Languages

Link to article, DOI:

10.1007/978-3-319-92408-3_8

Publication date:

2018

Document Version

Peer reviewed version

Link back to DTU Orbit

Citation (APA):

Kaminskas, L., \& Lluch Lafuente, A. (2018). Aggregation Policies for Tuple Spaces. In COORDINATION 2018: Coordination Models and Languages (pp. 181-199). Springer. Lecture Notes in Computer Science Vol. 10852 https://doi.org/10.1007/978-3-319-92408-3_8

\section{General rights}

Copyright and moral rights for the publications made accessible in the public portal are retained by the authors and/or other copyright owners and it is a condition of accessing publications that users recognise and abide by the legal requirements associated with these rights.

- Users may download and print one copy of any publication from the public portal for the purpose of private study or research.

- You may not further distribute the material or use it for any profit-making activity or commercial gain

- You may freely distribute the URL identifying the publication in the public portal

If you believe that this document breaches copyright please contact us providing details, and we will remove access to the work immediately and investigate your claim 


\title{
Aggregation policies for tuple spaces
}

\author{
Linas Kaminskas, Alberto Lluch Lafuente \\ DTU Compute, Technical University of Denmark \\ linaskmnsks@gmail.com, albl@dtu.dk
}

\begin{abstract}
Security policies are important for protecting digitalized information, control resource access and maintain secure data storage. This work presents the development of a policy language to transparently incorporate aggregate programming and privacy models for distributed data. We use tuple spaces as a convenient abstraction for storage and coordination. The language has been designed to accommodate well-known models such as $k$-anonymity and $(\varepsilon, \delta)$-differential privacy, as well as to provide generic user-defined policies. The formal semantics of the policy language and its enforcement mechanism is presented in a way that abstracts away from a specific tuple-space coordination language. To showcase our approach, an open-source software library has been developed in the Go programming language and applied to a typical coordination pattern used in aggregate programming applications.
\end{abstract}

Keywords: secure coordination, policy languages, privacy models, tuple spaces, aggregate programming

\section{Introduction}

Privacy is an essential part of society. With increasing digitalization of infrastructures grows their attack surface and the possibilities for abuse. It is therefore necessary to include privacy models that can scale with the complexity of those infrastructures and their software components, in order to protect information stored and exchanged while still ensuring information quality and availability. With EU GDPR regulation [19] being implemented in all EU countries, regulation on how data acquisition processes handle and distribute personal information becomes enforced. This affects software development processes and life cycles as security-by-design choices will need to be incorporated. Legacy systems will also be affected by GDPR compliance. With time, these legacy systems will need to be replaced, not only because of technological advancements, but also due to political and social demands for higher quality infrastructure. No matter the perspective, the importance of privacy-preserving data migration, mining and publication will remain relevant as society advances.

Aggregation, privacy and coordination Aggregate programming methods are used for providing privacy guarantees (e.g. by reducing the ability to distinguish individual data items), improving performance (e.g. by reducing storage size and communications) and even as the basis of emergent coordination 
paradigms (e.g. computational field and aggregate programming based on the field calculus [23,1] or the SMUC calculus [14]). Basic aggregation functions (e.g. sums, averages, etc.), do not offer enough privacy guarantees (e.g. against statistical attacks) to support the construction of trustworthy coordination systems. The risk is that less users will be willing to share their data and, as a consequence, the quality of infrastructures and services based on data aggregations may degrade. More powerful privacy protection systems are needed to reassure users and foster their participation with useful data. Fortunately, aggregationbased methods can be enhanced by using well-studied privacy models that allow policy makers to trade between privacy and data utility. We investigate in this work how such methods can be easily integrated in a coordination model such as tuple spaces, that in turn can be used as the basis of aggregation-based systems.

Motivational examples Of our main motivations is to address systems where users provide data in order to improve some services that they themselves may use. In such systems it is often the case that: (i) A user decides how much privacy is to be sacrificed when providing data. Data aggregation is performed according to a policy on their device and transmitted to a data collector. (ii) A data collector partitions data by some quality criterion. Aggregation is then performed on each partition and results are stored, while the received data may be discarded. (iii) A process uses the aggregated data, and shares results back to the users in order to provide a service.

A typical example of such systems are Intelligent Transport System (ITS), which exploit Geographic Information Systems (GIS) data from vehicles to provide better transportation services, e.g. increased green times at intersections, reduction of queue and congestion or exploration of infrastructure quality. As a real world example, bicycle GIS data is exploited by ITS systems to reduce congestion on bicycle paths, while maintaining individuals privacy. Figure 1 shows user positional data in different stages: (a) raw data as collected, (b) data after aggregating multiple trips,

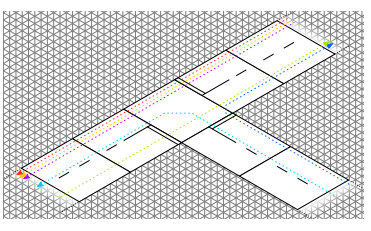

(a) Raw data

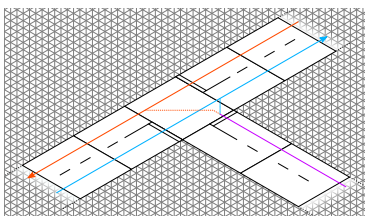

(b) Aggregated data

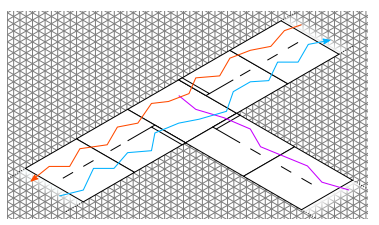

(c) Perturbed data

Fig. 1: Different stages of GIS data. and (c) aggregated data with addition of noise to protect privacy. This aggregated data can then be delivered back to the users, in order to support their decision making before more congestion occurs. Depending on the background knowledge and insights in a service, an adversary can partially or fully undo bare aggregation. By using privacy models and controlling aggregate functions, one can remove sensitive fields such as unique identifiers and device names, and add noise to give approximations of aggregation results. This gives a way to trade data accuracy in favor of privacy. 
Another typical example are selforganizing systems. Consider, for instance, the archetypal example of the construction of a distance field, identified in [2] as one of the basic self-organization building blocks for aggregate programming. The typical scenario in such systems is as follows. A number of devices and points-of-interests (PoI) are spread over a geographical area. The main aim of each device is to estimate the distance to the closest PoI. The resulting distributed mapping of devices into distances and possibly the next device on the shortest path, forms a computational field. This provides

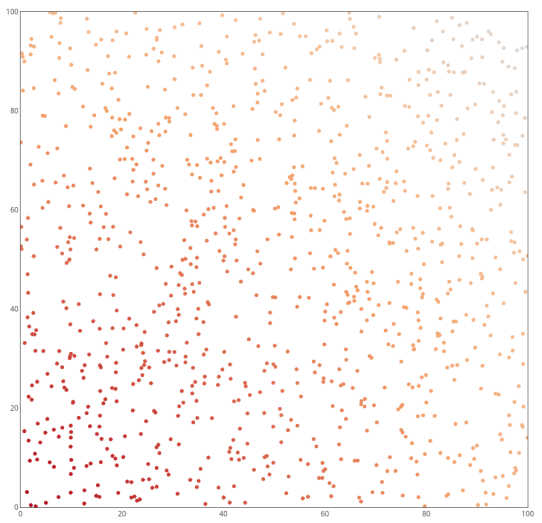

Fig. 2: A distance gradient field a basic layer for aggregate programming applications and coordinated systems, as in e.g. providing directions to PoIs. Figure 2 shows an example with the result of 1000 devices in an area with a unique PoI located at $(0,0)$, where each device is represented by a dot and whose color intensity is proportional to the computed distance. The computation of the field needs to be done in a decentralized way, since the range of communication of devices needs to be kept localized. The algorithm that the devices use to compute the field is based on data aggregations: a device iteratively queries the neighbouring devices for their information and updates its own information to keep track of the closest distance to a PoI. Initially, the distance $d_{i}$ of each device $i$ is set to the detected distance to the closest PoI, or to $\infty$ if no device is detected. At each iteration, a device $i$ updates its computed distance $d_{i}$ as follows. It gets from each neighbour $j$ its distance $d_{j}$, and then updates $d_{i}$ to be the minimum between $d_{i}$ or $d_{j}$ plus the distance from $i$ to $j$. In this algorithm, the key operation performed by the devices is an aggregation of neighbouring data, which may not offer sufficient privacy guarantees. For instance, the exact location of devices or their exact distance to a PoI could be inferred by a malicious agent. A simple case where this could be done is when one device and a PoI are in isolation. A more complex case could be if the devices are allowed to move and change their distances to a PoI gradually. By observing isolated devices and their interactions with neighbours, one could start to infer more about the behaviour of a device group.

Challenges Engineering of privacy mechanisms and embedding of these directly into large software systems is not a trivial task, and may be error prone. Therefore, it is crucial to separate privacy mechanisms from an application, in such a way that the privacy mechanisms can be altered without having to change the application logic. For example, Listing 1.1 shows a policy that a data hosting service will provide, and a program willing to use the data. The policy controls the aggregate query (aquery) of the program. It only allows to average (avg) some data and, in addition, it uses a add_noise function to the result before 


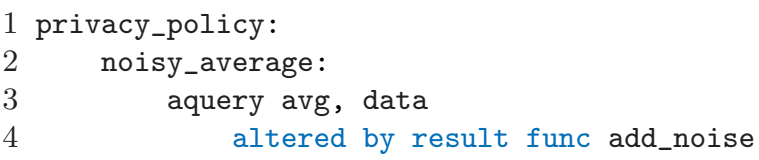

Listing 1.1: An aggregation policy that adds noise to average-based queries

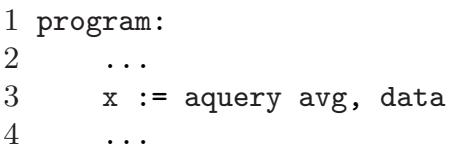

Listing 1.2: An aggregated query subject to the policy in Listing 1.1.

Fig. 3

an assignment in the program occurs. In this manner, a clear separation of logic can be achieved, and multiple queries of the same kind can be altered by the same policy. Furthermore, it allows policies to be changed at run-time, in order to adapt to changes in regulations or to optimize the implementation of a policy. Separation of concerns provides convenience for both developers and policy makers alike.

Contribution Our goal is to develop a tool-supported policy language providing access control for which well-studied privacy models, aggregate programming constructs, and coordination primitives could be used to provide non-intrusive data access in distributed applications. We wanted to focus on an interactive setting where data is dynamically produced, consumed and queried, instead of the traditional static data warehousing that privacy models implementations tend to address.

Our first contribution is a novel policy language in Section 2 to specify aggregation policies for tuple spaces. The choice of tuple spaces has been motivated by the need to abstract away from concrete data storage models and to address data-oriented coordination models. Our approach to the language provides a clean separation between policies that need to be enforced, and application logic that needs to be executed. The presentation abstracts away from any concrete tuple-based coordination language and we focus on aggregated versions of the traditional operations to add, retrieve and remove tuples.

Our second contribution (section 3) is a detailed description of how two well-studied privacy models such as $k$-anonymity and $(\varepsilon, \delta)$-differential privacy can be expressed in our language. For this purpose, those models (which are usually presented in a database setting) have been redefined in the setting of 
tuple spaces. To the authors knowledge, this is the first time that the definition of those models has been adapted to tuple spaces.

Our third and last contribution (section 4) is an open-source, publicly available implementation of the policy language and its enforcement mechanism in a tuple space library for the Go programming language, illustrated with an archetypal example of a self-organizing pattern used as basic block in aggregate programming approaches [2], namely the above presented computation of a distance gradient.

\section{A policy language for aggregations}

We start the presentation of our policy language by motivating the need of supporting and controlling aggregate programming primitives, and present a set of such primitives. We then move into the description of our policy language, illustrate the language through examples, and conclude the section with formal semantics.

Aggregate programming primitives The main computations we focus in this paper are aggregations of multiset of data items. As we have discussed in section 1, such computations are central to aggregate programming approaches. The main motivation is to control how such aggregations are performed: a data provider could want, for instance, to provide access to the average of a data set, but not to the data set or any of its derived forms. Traditional tuple spaces (e.g. those following the Linda model) do not support aggregations as first-class primitives: a user would need to extract all the data first and perform the aggregation during or after the extraction. Such a solution does not allow to control how aggregations are performed, and the user is in any case given access to an entire set of data items that needs to be protected. However, in databases, aggregate operators can be used in queries, providing thus a first-class primitive

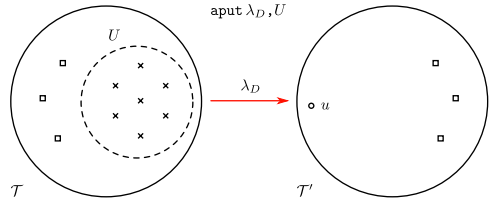

(a) Aggregated put: aput.

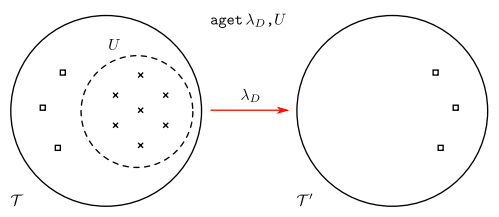

(b) Aggregated get: aget.

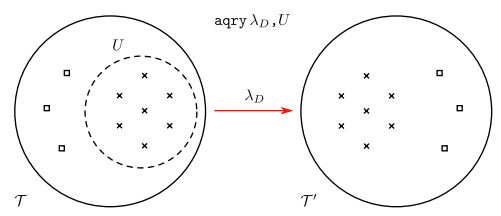

(c) Aggregated query: aqry. to perform aggregated queries, more amenable Fig. 4: Aggregation primitives.
for access control. A similar situation can be found in aggregate programming languages that provide functions to aggregate data from neighbouring components: the field calculus offers a nbr primitive to retrieve information about neighbouring devices and aggregation is to be performed on top of that, whereas the SMUC calculus is based on atomic aggregation primitives. 
We adapt such ideas to tackle the necessity of controlling aggregations in tuple spaces by proposing variants of the classical single-data operations put / out, get/in and qry/read. In particular, we extend them with an additional argument: an aggregation function that is intended to be applied to the multiset of all matched tuples. Typical examples of such functions would be averages, sums, minimum, concatenation, counting functions and so forth. While standard tuple space primitives allow to retrieve some or all tuples matching some template, the primitives we promote would allow to retrieve the aggregated version of all the matched tuples. More in detail, let's introduce the following aggregate programming primitives:

aqry $\lambda_{D}, U$ : This operation works similarly to an aggregated query in a database and provides an aggregated view of the data. In particular, it returns the result of applying the aggregation function $\lambda_{D}$ to all tuples that match the template $U$.

aget $\lambda_{D}, U$ : This operation is like aqry, but removes the matched data with template $U$.

aput $\lambda_{D}, U$ : This operation is like aget, but the result of the aggregation is introduced in the tuple space. It provides a common pattern used to atomically compress data.

It is worth to remark that such operations allow to replicate many of the common operations on tuple spaces. Indeed, the aggregation function could be, for instance, the multiset union (providing all the matched tuples) or a function that provides just one of the matched tuples (according to some deterministic or random function).

Syntax of the language The main concepts of the language are knowledge bases in the form of tuple spaces, policies for expressing how operations should be altered, and aggregate programming operators. The language itself can be embedded in any host coordination language, but the primary focus will be in expressing policies. The language is defined in a way that is reminiscent of a concrete syntax for a programming language. Although, the point is not to force a particular syntax, but to have a convenient abstraction for describing the policies themselves. Further, the language and aggregation policies do not force a traditional access control based model by only permitting or denying access to data: policies allow transformations thus giving different views on the same data. This gives a choice to a policy maker to control the accuracy of the information released to a data consumer. Subjects (e.g. users) and contextual (e.g. location) attributes are not part of our language, in order to keep the presentation focused on the key aspects of aggregate programming. Yet, the language could be easily extended to include these attributes.

The syntax of our aggregation policy language can be found in Table 1. Let $\Omega$ denote the types which are exposed by the host language and a type be $\tau \in \Omega$. For the sake of exposition we consider the simple case $\{$ int, float, string $\} \subseteq \Omega$. $\mathcal{T}$ is a knowledge base represented by a tuple space with a multiset interpretation, 


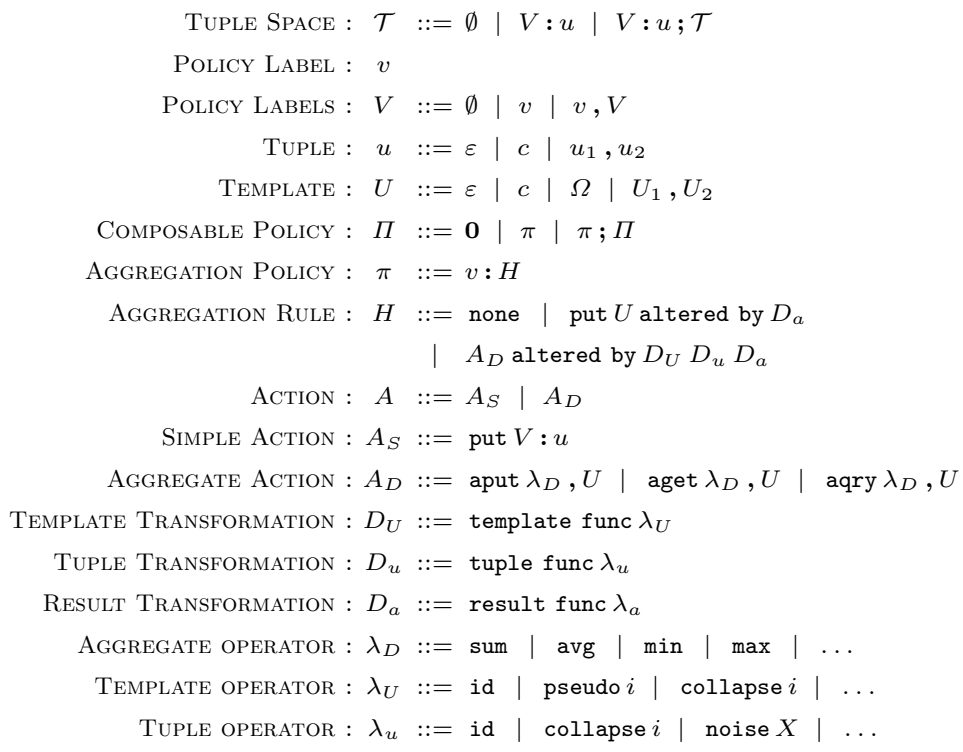

Table 1: Syntax for policies and aggregate programming operators.

where the order of tuples is irrelevant and multiple copies of the identical tuples are allowed. For $\mathcal{T}$, the language operator ; denotes the multiset union, $\backslash$ is the multiset difference and $\ominus$ is the multiset symmetric difference. $\mathcal{T}$ contains labelled tuples, i.e. tuples attached a set of labels, with each label identifying a policy. A tuple is denoted and generated by $u$, and an empty tuple is denoted by $\varepsilon$. Tuples may be primed $u^{\prime}$ or stared $u_{\star}$ to distinguish between different types of tuples. The type of a tuple $u$ is denoted by $\tau_{u}=\tau_{u_{1}} \times \tau_{u_{2}} \times \ldots \times \tau_{u_{n}}$. In Section 3 , individual tuple fields will be needed, and hence we will be more explicit and use $u=\left(u_{1}, \ldots, u_{i}, \ldots, u_{n}\right)$, where $u_{i}$ denotes the $i^{\text {th }}$ tuple field with type $\tau_{u_{i}}$. When dealing with a multiset of tuples of type $\tau_{u}$ (e.g. a tuple space), the type $\tau_{u}^{*}$ will be used. For a label set $V$, a labelled tuple is denoted by $V: u$. Similarly as for a tuple, the empty labelled tuple is denoted by $\varepsilon$. A label serves as a unique attribute when performing policy selection based on an action A. A template $U$ can contain constants $c$ and types $\tau \in \Omega$ and is used for pattern matching against a $u \in \mathcal{T}$. As with tuples, we shall be explicit with template fields when necessary and use $U=\left(U_{1}, \ldots, U_{i}, \ldots, U_{n}\right)$, where $U_{i}$ denotes the $i^{\text {th }}$ template field with type $\tau_{U_{i}}$. There are three main aggregation actions derived from the classical tuple space operations (put, get, qry), namely: aput, aget and aqry. All operate by applying an aggregate operator $\lambda_{D}$ on tuples $u \in \mathcal{T}$ that matches $U$. Aggregate functions $\lambda_{D}$ have a functional type $\lambda_{D}: \tau_{u}^{*} \rightarrow \tau_{u^{\prime}}$ and are used to aggregate tuples of type $\tau_{u}$ into a tuple of type $\tau_{u^{\prime}}$. The composable policy $\Pi$ is a list of policies that contain aggregation policies $\pi$. An aggregation $\pi$ is defined by a policy label $v$ and an aggregation rule $H$, where $v$ is used as an identifier for $H$. An aggregation rule $H$ describes how an action $A$ is altered either by a template transformation $D_{U}$, a tuple transformation $D_{u}$, and a result transformation $D_{a}$, or not at all by none. A template transformation 
$D_{U}$ is defined by a template operator $\lambda_{U}: \tau_{U} \rightarrow \tau_{U^{\prime}}$, and can be used for e.g. hiding sensitive attributes or to adapt the template from the public format of tuples to the internal format of tuples. A tuple transformation $D_{u}$ is defined by a tuple operator $\lambda_{u}: \tau_{u} \rightarrow \tau_{u^{\prime}}$. This allows to apply additional functions on a matched tuple $u$, and can be used e.g. for doing sanitization, addition of noise or approximating values, before performing the aggregate operation $\lambda_{D}$ on the matched tuples A result transformation $D_{a}$ is defined by a tuple operator $\lambda_{a}: \tau_{u^{\prime}} \rightarrow \tau_{u^{\prime \prime}}$. The arguments of $\lambda_{a}$ are the same as for tuple transformations $\lambda_{u}$, except the transformation is applied on an aggregated tuple. This allows for coarser control, say, in case a transformation on all the matched tuples is computationally expensive or if simpler policies are enough.

Examples and comparison with a database Observe that $\lambda_{D}$ and any of the aggregation actions in $A$ can provide all of the aggregate functions found in commercial databases, but with the flexibility of exactly defining how this is performed in the host language itself. The motivation for doing this comes from the fact that: (i) there is tendency for database implementations to provide non-standardized functionalities, introducing software fragmentation when swapping technologies, (ii) user-defined aggregate functions are often defined in a different language from the host language. In our approach, by allowing to directly express both the template for the data needed and aggregate functionality in the host language, helps reducing the programming complexity and improves readability, as the intended aggregation is expressed explicitly and in one place. Moreover, the usage of templates allows to specify the view of data at different granularity levels. For instance, in our motivational example on GIS data, one could be interested in expressing:

1. Field granularity where $U$ contains concrete values only, but access is provided to some fields only. Listing 1.3 shows how to allow access to a specific data source by using $U=$ ("devices", "gps", "accelerometer", "gyroscope") as a template of concrete devices. Here, id is the identity function, first is an aggregation function which returns the first matched tuple, and nth 2 selects the second field of the tuple.

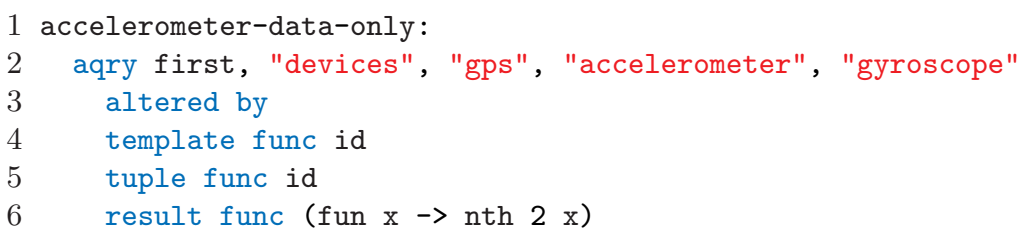

Listing 1.3: Example of field granularity policy.

2. Tuple granularity where $U$ contains concrete values and all fields are provided. Listing 1.4 shows how a policy can provide access to a specific trip. In this case, it is specified by a trip type and trip identifier $U=$ ("bike-ride", 1).

1 single-ride: 


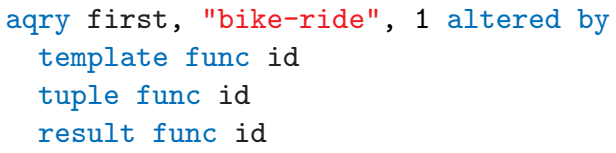

Listing 1.4: Example of tuple granularity policy.

3. Mixed granularity where $U$ contains a mix of concrete values and types. Listing 1.5 shows how this could be used to protect user coordinates expressed as a triplet of float's encoding latitude, longitude, elevation while allowing a certain area. In this case, the copenhagen area is exposed, and computation of the average elevation with avg is permitted.

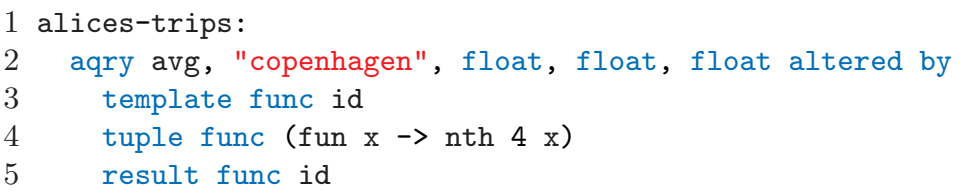

Listing 1.5: Example of mixed granularity policy.

4. Tuple-type granularity where $U$ contains only types. Listing 1.6 shows how this could be used to count how many points there are in each discretized part of a map, where area maps coordinates into areas.

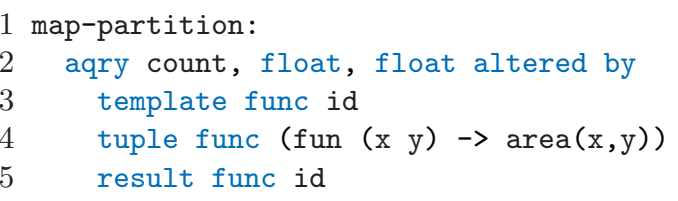

Listing 1.6: Example of tuple-type granularity policy.

With respect to databases, the aforementioned granularities correspond to: 1 . cell level, 2. single row level, 3. multiple row level, and 4. table level. Combined with a user-defined aggregate function $\lambda_{D}$ and transformations $D_{U}, D_{u}$ and $D_{a}$, one can provide many different views of a tuple space in a concise manner.

Formal semantics Before presenting the formal semantics, we provide a graphical and intuitive presentation using $A=\operatorname{aqry} \lambda_{D}, U$ and some $\mathcal{T}$ and $\Pi$ as an example shown in Figure 5. The key idea is: 1. Given some action $A$, determine the applicable policy $\pi$. There can be multiple matches in $\Pi$; 2 . extract the first-matching policy $\pi$ with some label $v ; 3$. extract template, tuple and result operators from transformations $D_{U}, D_{u}$, and $D_{a}$ respectively; 4 . extract the aggregate operator $\lambda_{D}$ and apply $U^{\prime}=\lambda_{U}(U) ; 5$. based on the tuples $V: u$ from $\mathcal{T}$ that match $U^{\prime}$ and have $v \in V$ : perform tuple transformation with $\lambda_{u}$, aggregation with $\lambda_{D}$, and result transformation with $\lambda_{a}$.

The formal operational semantics of our policy enforcement mechanism is described by the set of inference rules in table 2 , whose format is

$$
\begin{array}{cccc}
P_{1} \quad \ldots \quad P_{i} & \ldots & P_{n} \\
\mathcal{T}, \Pi \vdash A \rightarrow \mathcal{T}^{\prime}, \Pi \triangleright R
\end{array}
$$




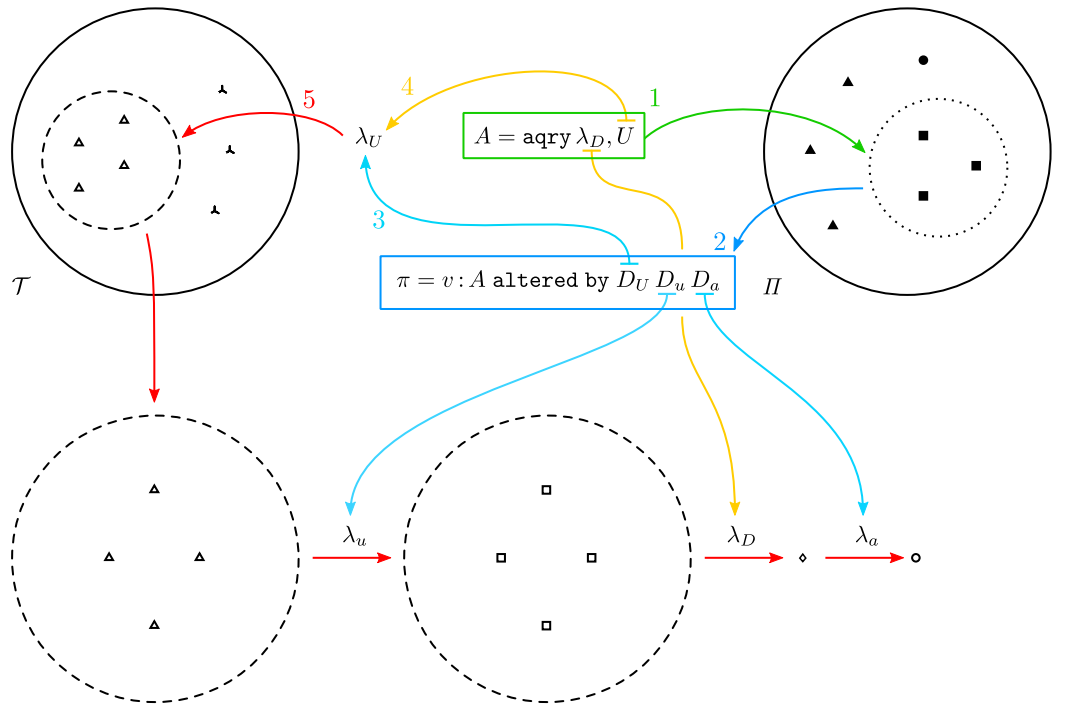

Fig. 5: Semantics of an aggregate action $A$ given an applicable policy $\pi$.

where $P_{1}, \ldots, P_{n}$ are premises, $\mathcal{T}$ is a tuple space subject to $\Pi, A$ is the action subject to control, and the return value (if any) is modelled by $\triangleright R$. The return value may then be consumed by the host language. The absence of a return value denotes that no policy was applicable.

The semantics for applying a policy that matches an aggregate action aput $\lambda_{D}, U$, aget $\lambda_{D}, U$ and aqry $\lambda_{D}, U$ is respectively defined by rules AGG-PUT-ApPLY, AgG-Get-Apply and AgG-Query-Apply. For performing put $V: u$, PutAPPLY is used. All three rules apply such transformation and differ only in that aget and aput modify the tuple space. A visual representation of the semantics of aput $\lambda_{D}, U$, aget $\lambda_{D}, U$ and aqry $\lambda_{D}, U$ can be seen in Figure 5 . The premises of the rules include conditions to ensure that the right operation is being captured and a decomposition of how the operation is transformed by the policy. In particular, the set $\mathcal{T}_{1}$ represents the actually matched tuples (after transforming the template) and $\mathcal{T}_{2}$ is the actual view of the tuple space being considered (after applying the tuple transformations to $\mathcal{T}_{1}$ ). It is on $\mathcal{T}_{2}$ that the user-defined aggregation $\lambda_{D}$ is applied, and then the result transformation $\lambda_{a}$ is applied to provide the final result $u_{a}$. Rules named Unmatched, PriorityLeft, Priority-Left, and Priority-Unavallable take care of scanning the policy as list. It is up to the embedding in an actual host language to decide what to do with the results. For example, in our implementation, if the policy enforcement yields no result, the action is simply ignored. 


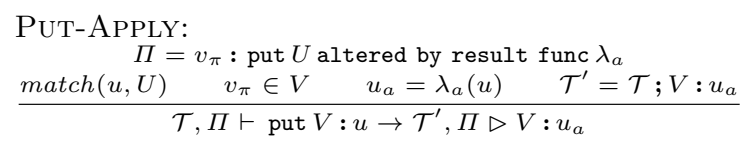

AGG-Put-ApPly:

$\Pi=v_{\pi}$ : aput $\lambda_{D}, U^{\prime}$ altered by template func $\lambda_{U}$ tuple func $\lambda_{u}$ result func $\lambda_{a}$ $\operatorname{match}\left(U, U^{\prime}\right) \quad \mathcal{T}_{1}=\left\{V: u \in \mathcal{T} \mid \operatorname{match}\left(u, \lambda_{U}(U)\right) \wedge v_{\pi} \in V\right\} \quad \mathcal{T}^{\prime}=\mathcal{T} \backslash \mathcal{T}_{1}$ $\underline{\mathcal{T}_{2}=\left\{V: \lambda_{u}(u) \mid V: u \in \mathcal{T}_{1}\right\} \quad u_{a}=\lambda_{a}\left(\lambda_{D}\left(\left\{u \mid V: u \in \mathcal{T}_{2}\right\}\right)\right) \quad \mathcal{T}^{\prime \prime}=\mathcal{T}^{\prime} ;\left\{v_{\pi}\right\}: u_{a}}$

AGG-GET-APPLY:

$\Pi=v_{\pi}$ : aget $\lambda_{D}, U^{\prime}$ altered by template func $\lambda_{U}$ tuple func $\lambda_{u}$ result func $\lambda_{a}$ $\operatorname{match}\left(U, U^{\prime}\right) \quad \mathcal{T}_{1}=\left\{V: u \in \mathcal{T} \mid \operatorname{match}\left(u, \lambda_{U}(U)\right) \wedge v_{\pi} \in V\right\}$

$\frac{\mathcal{T}^{\prime}=\mathcal{T} \backslash \mathcal{T}_{1} \quad \mathcal{T}_{2}=\left\{V: \lambda_{u}(u) \mid V: u \in \mathcal{T}_{1}\right\} \quad u_{a}=\lambda_{a}\left(\lambda_{D}\left(\left\{u \mid V: u \in \mathcal{T}_{2}\right\}\right)\right)}{\mathcal{T}, \Pi \vdash \operatorname{aget} \lambda_{D}, U \rightarrow \mathcal{T}^{\prime}, \Pi \triangleright\left\{v_{\pi}\right\}: u_{a}}$

AgG-QUERY-APPLY:

$\Pi=v_{\pi}$ : aqry $\lambda_{D}, U^{\prime}$ altered by template func $\lambda_{U}$ tuple func $\lambda_{u}$ result func $\lambda_{a}$ $\operatorname{match}\left(U, U^{\prime}\right) \quad \mathcal{T}_{1}=\left\{V: u \in \mathcal{T} \mid \operatorname{match}\left(u, \lambda_{U}(U)\right) \wedge v_{\pi} \in V\right\}$ $\mathcal{T}_{2}=\left\{V: \lambda_{u}(u) \mid V: u \in \mathcal{T}_{1}\right\} \quad u_{a}=\lambda_{a}\left(\lambda_{D}\left(\left\{u \mid V: u \in \mathcal{T}_{2}\right\}\right)\right)$ $\mathcal{T}, \Pi \vdash$ aqry $\lambda_{D}, U \rightarrow \mathcal{T}, \Pi \triangleright\left\{v_{\pi}\right\}: u_{a}$

UNMATCHED:

$\Pi=v_{\pi}:$ none $\vee\left(\Pi=v_{\pi}: A_{2}\right.$ altered by $\left.D_{U} D_{u} D_{a} \wedge A_{1} \neq A_{2}\right) \vee \Pi=\mathbf{0}$ $\mathcal{T}, \Pi \vdash A_{1} \rightarrow \mathcal{T}, \Pi$

PRIORITY-RIGHT:

$$
\begin{array}{lr}
\mathcal{T}, \Pi_{1} \vdash A \rightarrow \mathcal{T}, \Pi_{1} & \text { PRIORITY-LEFT: } \\
\mathcal{T}, \Pi_{1} \vdash A \rightarrow \mathcal{T}
\end{array}
$$

$$
\begin{array}{cc}
\mathcal{T}, \Pi_{1} \vdash A \rightarrow \mathcal{T}, \Pi_{1} & \mathcal{T}, \Pi_{1} \vdash A \rightarrow \mathcal{T}^{\prime}, \Pi_{1}^{\prime} \triangleright V: u \\
\hline \mathcal{T}, \Pi_{2} \vdash A \rightarrow \mathcal{T}^{\prime}, \Pi_{2}^{\prime} \triangleright V: u & \frac{\mathcal{T}, \Pi_{1} ; \Pi_{2} \vdash A \rightarrow \mathcal{T}^{\prime}, \Pi_{1}^{\prime} ; \Pi_{2} \triangleright V: u}{\mathcal{T}, \Pi_{1} ; \Pi_{2} \vdash A \rightarrow \mathcal{T}^{\prime}, \Pi_{1} ; \Pi_{2}^{\prime} \triangleright V: u}
\end{array}
$$

Priority-UNAVAILABLE:

$\frac{\mathcal{T}, \Pi_{1} \vdash A \rightarrow \mathcal{T}, \Pi_{1} \quad \mathcal{T}, \Pi_{2} \vdash A \rightarrow \mathcal{T}, \Pi_{2}}{\mathcal{T}, \Pi_{1} ; \Pi_{2} \vdash A \rightarrow \mathcal{T}, \Pi_{1} ; \Pi_{2}}$

Table 2: Semantics for action $A$ under $\Pi$ including the semantics format.

\section{Privacy models}

The design of our language has been driven by inspecting a variety of privacy models, first and foremost $k$-anonymity and $(\varepsilon, \delta)$-differential privacy. We show in this section how those models can be adopted in our approach. The original definitions have been adapted from databases to our tuple space setting.

$\boldsymbol{k}$-anonymity The essential idea of $\boldsymbol{k}$-anonymity $[20,22,15]$ is to provide anonymity guarantees beyond hiding sensitive fields by ensuring that, when information on a data set is released, every individual data item is indistinguishable from at least $k-1$ other data items. In our motivational examples, for instance, this could be helpful to protect the correlation between devices and their distances from an attacker that can observe the position and number of devices in a zone and can obtain the list of distances within a zone through a query. $k$-anonymity is often defined for tables in a database, here it shall be adapted to templates 
$U$ instead. We start by defining $k$-anonymity as a property of $\mathcal{T}$ : roughly, $k$ anonymity requires that every tuple $u$ cannot be distinguished from at least $k-1$ other tuples. Distinguishability of tuples is captured by an equivalence relation $=_{t}$. Note that $=_{t}$ is not necessarily as strict as tuple equality: two tuples $u$ and $u^{\prime}$ may be different but equivalent, in the sense that they can be related exactly by the same, and possibly external, data. In our setting, $k$-anonymity is formalized as follows.

Definition 1 ( $k$-anonymity). Let $k \in \mathbb{N}^{+}, \mathcal{T}$ be a multiset of tuples, and let ${ }_{t}$ be an equivalence relation on tuples. $\mathcal{T}$ has $k$-anonymity for $=_{t}$ if:

$$
\forall u \in \mathcal{T} .\left|\left\{u^{\prime} \in \mathcal{T}_{U} \mid u^{\prime}={ }_{t} u\right\}\right| \geq k
$$

In other words, the size of the non-empty equivalence classes induced by $=_{t}$ is at least $k$. We say that a multiset of tuples $\mathcal{T}$ has $k$-anonymity if $\mathcal{T}$ has $k$ anonymity for $=_{t}$ being tuple equality (the finest equivalence relation on tuples). $k$-anonymity is not expected to be a property of the tuple space itself, but of the release of data provided by the operations aqry, aget and aput. In particular, we say that $k$-anonymity is provided by a policy $\Pi$ on a tuple space $\mathcal{T}$ when for every query based on the above operations the released result $u_{a}$ (cf. Figure 5) has $k$-anonymity. Note that this does only make sense if the result $u_{a}$ is a multiset of tuples, which could be the case when the aggregation function is a multiset operation like multiset union. Policies can be used to enforce $k$-anonymity on specific queries. Consider for instance the previously mentioned example of the attacker trying to infer information about distances and positions of devices. Assume the device information is stored in tuples $(x, y, i, j, d)$ where $(x, y)$ are actual coordinates of the devices, $(i, j)$ represents the zone in the grid and $d$ is the computed distance to the closest PoI. Suppose further that we want to provide access to a projection of those tuples by hiding the actual positions and providing zone and distance information. Hiding the positions is not enough and we want to to provide 2 -anonymity on the result. We can do so with the following policy:

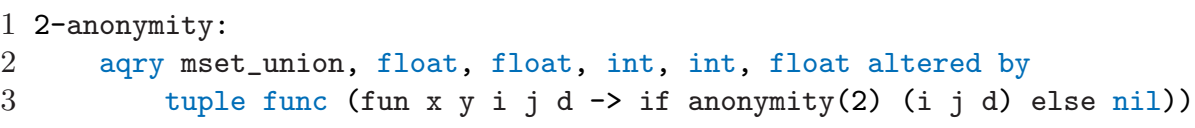

Listing 1.7: Example of $k$-anonymity for $k=2$.

where anonymity $(\mathrm{k})$ checks $k$-anonymity on the provided view $\mathcal{T}_{2}$ (cf. Figure 5 ), according to Definition 1. Basically, the enforcement of the policy will ensure that we provide the expected result, if in each zone there are at least two devices with the same computed distance, otherwise the query produces the empty set.

$(\varepsilon, \delta)$-differential privacy Differential privacy techniques [7] aim at protecting against attackers that can perform repeated queries with the intention of inferring information about the presence and/or contribution of single data item in a data set. The main idea is to add controlled noise to the results of queries 
so to reduce the amount of information that such attackers would be able to obtain. Data accuracy is hence sacrificed for the sake of privacy. For instance, in the motivational example of the distance gradient, differential privacy can be used to the approximate the result of the aggregations performed by the gradient computation. This is done in order to minimize leakage about the actual positions and distance of each neighbouring device. Differential privacy is a property of a randomized algorithm, where the data set is used to give enough state information in order to increase indistinguishably. Randomization arises from privacy protection mechanisms based on e.g. sampling and adding randomly distributed noise. The property requires that performing a query for all possible neighbouring subsets of some data set, the addition (or removal) of a single data item produces almost indistinguishable results. Differential privacy is often presented in terms of histogram representations of databases not suitable for our purpose. We present in the following a reformulation of differential privacy for our setting. Let $\mathbf{P}[\mathcal{A}(\mathcal{T}) \in S]$ denote the probability that the output $\mathcal{A}(\mathcal{T})$ of a randomized algorithm $\mathcal{A}$ is in $S$ when applied to $\mathcal{T}$, where $S \subseteq \mathbf{R}(\mathcal{A})$ and $\mathbf{R}(\mathcal{A})$ is the codomain of $\mathcal{A}$. In our setting $\mathcal{A}$ should be seen as the execution of an aggregated query, and that randomization arises from random noise addition. $(\varepsilon, \delta)$-differential privacy in our setting is then defined as the following property.

Definition $2((\varepsilon, \delta)$-differential privacy). Let $\mathcal{A}$ be a randomized algorithm, $\mathcal{T}$ be a tuple space, e be Euler's number, and $\varepsilon$ and $\delta$ be real numbers. $\mathcal{A}$ satisfies $(\varepsilon, \delta)$-differential privacy if and only if for any two tuple spaces $\mathcal{T}_{1} \subseteq \mathcal{T}$ and $\mathcal{T}_{2} \subseteq \mathcal{T}$ such that $\left\|\mathcal{T}_{1} \ominus \mathcal{T}_{2}\right\|_{\tau_{u}} \leq 1$, and for any $S \subseteq \mathbf{R}(\mathcal{A})$, the following holds:

$$
\mathbf{P}\left[\mathcal{A}\left(\mathcal{T}_{1}\right) \in S\right] \leq e^{\varepsilon} \cdot \mathbf{P}\left[\mathcal{A}\left(\mathcal{T}_{2}\right) \in S\right]+\delta
$$

Differential privacy can be enforced by policies that add a sufficient amount of random noise to the result of the queries. There are several noise addition algorithms that guarantee differential privacy. A common approach is based on the global sensitivity of data set for an operation and a differentially private mechanism which uses the global sensitivity to add the noise. Global sensitivity measures the largest possible distance between neighbouring subsets (i.e. differing in exactly one tuple) of a tuple space, given an operation. The differentially private mechanism uses this measure to distort the result when the operation is applied. To define a notion of sensitivity in our setting, assume that for every basic type $\tau$ there is a norm function $\|\cdot\|: \tau_{u} \rightarrow \mathbb{R}$ which maps every tuple into a real number. This is needed in order to define a notion of difference between tuples. We are now ready to define a notion of sensitivity for a given aggregate operator $\lambda_{D}$.

Definition 3 (Sensitivity). Let $\mathcal{T}$ be a tuple space, $\lambda_{D}: \tau_{u^{\prime}} \rightarrow \tau_{u_{\star}}$ be an aggregation function, and $p \in \mathbb{N}^{+}$. The $p^{\text {th }}$-global sensitivity $\Delta_{p}$ of $\lambda_{D}$ is defined as:

$$
\Delta_{p}\left(\lambda_{D}\right)=\max _{\substack{\forall \mathcal{T}_{1}, \mathcal{T}_{2} \subseteq \mathcal{T} \\ \mathcal{T}_{1} \ominus \mathcal{T}_{2}=1}} \sqrt[p]{\sum_{i \in\left\{0, . .,\left|\tau_{u^{\prime}}\right|\right\}}\left|\left\|\lambda_{D}\left(\mathcal{T}_{1}\right)_{i}\right\|-\left\|\lambda_{D}\left(\mathcal{T}_{2}\right)_{i}\right\|\right|^{p}}
$$


Roughly, Equation (1) is expressing that the sensitivity scale of an aggregate operator is determined by the largest value differences between all fields of the aggregated tuples. The global sensitivity can then be used to introduce Laplace noise according to the well-known Laplace mechanism, which provides $(\varepsilon, 0)$ differential privacy.

Definition 4 (Laplace noise addition). Let $\mathcal{T}$ be a tuple space, $\lambda_{D}: \tau_{u}^{*} \rightarrow$ $\tau_{u^{\prime}}$ be an aggregation function, $\oplus: \tau_{u^{\prime}} \times \tau_{u^{\prime}} \rightarrow \tau_{u^{\prime}}$ be an addition operator for type $\left.\left.\tau_{u^{\prime}}, \varepsilon \in\right] 0,1\right], p \in \mathbb{N}^{+}$, and $\boldsymbol{Y}=\left(Y_{1}, \ldots, Y_{i}, \ldots, Y_{n}\right)$ be a tuple of random variables that are independently and identically distributed according to the Laplace distribution $Y_{i} \sim \mathcal{L}\left(0, \Delta_{p}\left(\lambda_{D}\right) / \varepsilon\right)$. The Laplace noise addition function laplace ${\mathcal{T}, \lambda_{D}, \varepsilon}$ is defined by:

$$
\text { laplace }_{\mathcal{T}, \lambda_{D}, \varepsilon}(u)=u \oplus \boldsymbol{Y}
$$

Note that the function is parametric with respect to the noise addition operator $\oplus$. For numerical values $\oplus$ is just ordinary addition. In general, for $\oplus$ to be to be meaningful, one has to define it for any type. For complex types such as strings, structures or objects this is not trivial, and either one has to have a well-defined $\oplus$ or other mechanisms should be considered for complex data types.

Consider again our motivational example of distance gradient computation, we can define a policy to provide differential privacy on the aggregated queries of each round of the computation as follows:

1 edp:

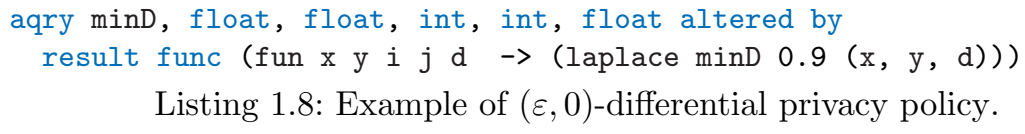

The policy controls queries aiming at retrieving the information $(x, y)$ coordinates, $(i, j)$ zone and distance $d$ of the device that is closest to a PoI, obtained by the aggregation function minD. The query returns only the coordinates of such device and its distance, after distorting them with Laplace noise by function laplace, implemented according to Definition 4 (with the tuple space being the provided view $\mathcal{T}_{2}$, cf. Figure 5 ).

More in general, the enforcement of policies of the form

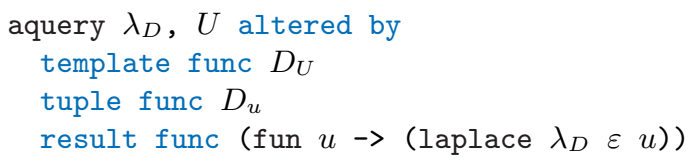

Listing 1.9: Schema for $(\varepsilon, 0)$-differential privacy policies.

provides $(\varepsilon, 0)$-differential privacy on the view of the tuple space ( $\mathrm{cf}$. $\mathcal{T}_{2}$ in Figure 5) against aggregated queries based on the aggregation function $\lambda_{D}$.

\section{Aggregation policies at work}

To showcase the applicability of our approach to aggregate computing applications, we describe in this section a proof-of-concept implementation of our policy 
language and its enforcement mechanism in a tuple space library (cf. Section 4.1), and the implementation of one of the archetypal self-organizing building blocks used in aggregate programming, namely the computation of a distance gradient field, that we use also to benchmark the library (cf. Section 4.2).

\subsection{Implementation of a proof-of-concept library}

The open-source library we have implemented is available for download, installation and usage at https://github.com/pSpaces/goSpace. The main criteria for choosing Go was that it provides a reasonable balance between language features and minimalism needed for a working prototype. Features that were considered important included concurrent processes, a flexible reflection system and a concise standard library. The goSpace project was chosen because it provided a basic tuple space implementation, and had the fundamental features, such as addition, retrieval and querying of tuples based on templates, and it also provides derived features such as retrieval and querying of multiple tuples. Yet, goSpace itself was modified in order to provide additional features needed for realizing the policy mechanism. One of the key features of the implementation is a form of code mobility that allows to transfer functions across different tuple spaces. This was necessary to serve as a foundation for allowing user-defined aggregate functions across multiple tuple spaces. Further, the library was implemented to be slightly more generic than what is given in Section 2 and can in principle be applied to other data structures beyond tuple spaces and aggregation operators on tuple space. Currently, our goSpace implementation supports policies for the actions aput, aget and aqry but it can be easily extended to support additional operations.

\subsection{Protecting privacy in a distance gradient}

We have implemented the case study of the distance field introduced in Section 1 as a motivational example. In our implementation, the area where devices and PoIs are placed, is discretized as a grid of zones; each device and PoI has a position and is hence located in a zone. The neighbouring relation is given by the zones: two devices are neighbours if their zones are incident. Devices can only detect PoIs in their own zone and devices cannot communicate directly with each other: they use a tuple space to share their information. Each device publishes in the tuple space their information (position, zone and computed distance) labelled with a privacy policy. The aggregation performed in each round uses the aqry operation with an aggregation function that selects the tuple with the smallest distance to a PoI.

Different policies can be considered. The identity policy would simply correspond to the typical computation of the field as seen in the literature. Basically, all devices and the tuple space are considered to be trustworthy and no privacy guarantees are provided. Another possibility would be to consider that devices, and other agents that want to exploit the field, cannot be fully trusted with respect to privacy issues. A way to address this situation would be to consider 


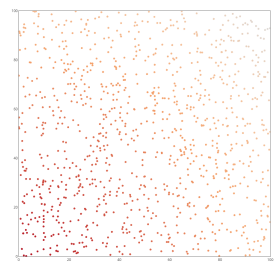

(a) noise 0.01

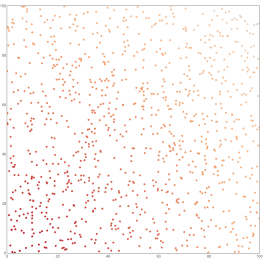

(b) noise 0.1

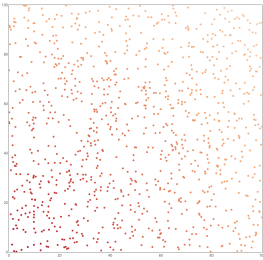

(c) noise 0.2

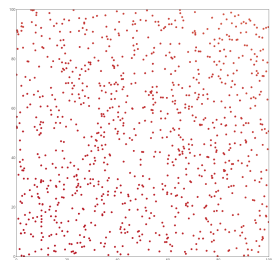

(d) noise 1.0

Fig. 6: Distance gradients with aggregation policies based on noise addition.

policies that hide or distort the result of the aggregated queries used in each round.

We have performed several experiments with our case study and we have observed, as expected, that such polices may affect accuracy (due to noise addition) and performance (due to the overhead of the policy enforcement mechanism). Some results are depicted in Figure 2, Figure 6 and Figure 7. In particular the figures show experiments for a scenario with 1000 devices and a discretization of the map into a $100 \times 100$ grid. Figure 2 shows the result where data is not protected but is provided as-it-is, while Figure 6 shows results that differ in the amount of noise added to the distance ob-

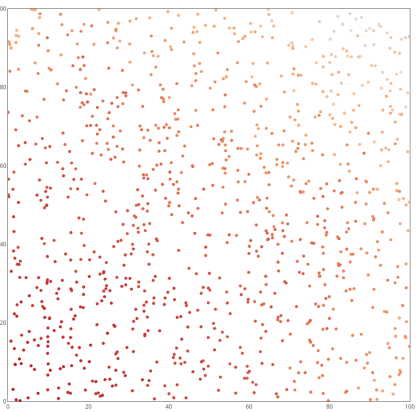

Fig. 7: Gradient with noise tained from the aggregated queries. This is regulated by a parameter $x$ so that the noise added is drawn from a uniform distribution in $[-x * d, x * d]$, where $d$ is the diameter of each cell of the grid (actually $\sqrt{10 * 10}$ ). In the figures, each dot represents a device and the color intensity is proportional to the distance to the PoI, which is placed at $(0,0)$. Highest intensity corresponds to distance 0 , while lowest intensity corresponds to the diameter of the area $(\sqrt{200})$. The results with more noise (bottom-right of Figure 6) make it evident how noise can affect data accuracy: the actual distance seems to be the same for all nodes. However, Figure 6, which shows the same data but where the color intensity goes from 0 to the maximum value in the field, reveals that the price paid for providing more privacy does not affect much the field: the gradient towards the PoI is still recognizable.

\section{Conclusion}

We have designed and implemented a policy language which allows to succinctly express and enforce well-understood privacy models in the syntactic category such as $k$-anonymity, and in the semantic category such as $(\varepsilon, \delta)$-differential privacy. Aggregate operations and templates defined for a tuple space were used 
to give a useful abstraction for aggregate programming. Even if not shown here, our language allows to express additional syntactic privacy models such as $\ell$ diversity [15], $t$-closeness [13,21] and $\delta$-presence [8]. Our language does not only allow to adopt the above mentioned privacy models but it is flexible enough to specify and implement additional user-defined policies. The policy language and its enforcement mechanism have been implemented in a publicly available tuple space library. The language presented here has been designed with minimality in mind, and with a focus on the key aspects related to aggregation and privacy protection. Several aspects of the language can be extended, including richer operations to compose policies and label tuples, user-dependent and contextaware policies, tuple space localities and polyadic operations (e.g. to aggregate data from different sources as usual in aggregate computing paradigms). We believe that approaches like ours are fundamental to increase the security and trustworthiness of distributed and coordinated systems.

Related work We have been inspired by previous works that enriched tuple space languages with access control mechanism, in particular SCEL [6,16] and KLAIM [11,3]. We have also considered database implementations with access control mechanisms amenable for the adoption of privacy models. For example, [5] discusses the development strategies for FBAC (fine-grained access control) frameworks in NoSQL databases and showcases applications for MongoDB, the Qapla policy framework [18] provides a way to manipulate DBMS queries at different levels of data granularity and allows for transformations and query rewriting similar to ours, and PINQ [17] uses LINQ, an SQL-like querying syn-

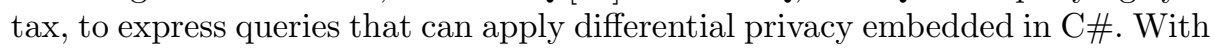
respect to databases our approach provides a different granularity to control operations, for instance our language allows to easily define template-dependent policies. Our focus on aggregate programming has been also highly motivated by the emergence of aggregate programming and its application to domains of increasing interest such as the IoT [1]. As far as we know, security aspects of aggregate programming are considered only in [4] where the authors propose to enrich aggregate programming approaches with trust and reputation systems to mitigate the effect of malicious data providers. Those considerations are related to data integrity and not to privacy. Another closely related work is [9] where the authors present an extension to a tuple space system with privacy properties based on cryptography. The main difference with respect to our work is in the different privacy models and guarantees considered.

Future Work One of the main challenges of current and future privacy protection systems for distributed systems, such as the one we have presented here, is their computational expensiveness. We plan to carry out a thorough performance evaluation of our library. We plan in particular to experiment with respect to different policies and actions. It is well known that privacy protection mechanism may be expensive and finding a right trade-off is often applicationdependent. Part of the overhead in our library is due to the preliminary status 
of our implementation where certain design aspects have been done in a naive manner to prioritize rapid prototyping over performance optimizations, e.g. use of strong cryptographic hashing, use of standard library concurrent maps and redundancies in some data structures. This makes room for improvement and we expect that the performance of our policy enforcement mechanism will be significantly improved. More in general, finding the optimal $k$-anonymity is an NP-hard problem. There is however room for improvements. For instance, [12] provides an approximation algorithm. This algorithm could be adapted if enforcement is needed. An online differentially private algorithm, namely private multiplicative weights algorithm, is given in [7]. Online algorithms are worth of investigation since interactions with $\mathcal{T}$ are inherently online. Treatment of functions and functional data in differential privacy setting can be found [10]. We are currently investigating online efficient algorithms to improve the performance of our library.

\section{References}

1. J. Beal, D. Pianini, and M. Viroli. Aggregate programming for the internet of things. Computer, 48(9):22-30, 2015.

2. J. Beal and M. Viroli. Building blocks for aggregate programming of self-organising applications. In Eighth IEEE International Conference on Self-Adaptive and SelfOrganizing Systems Workshops, SASOW 2014, London, United Kingdom, September 8-12, 2014, pages 8-13. IEEE Computer Society, 2014.

3. G. Bruns and M. Huth. Access-control policies via belnap logic: Effective and efficient composition and analysis. Csf 2008: 21st Ieee Computer Security Foundations Symposium, Proceedings, pages 163-+, 2008.

4. R. Casadei, A. Aldini, and M. Viroli. Combining trust and aggregate computing. In A. Cerone and M. Roveri, editors, Software Engineering and Formal Methods - SEFM 2017 Collocated Workshops: DataMod, FAACS, MSE, CoSim-CPS, and FOCLASA, Trento, Italy, September 4-5, 2017, Revised Selected Papers, volume 10729 of Lecture Notes in Computer Science, pages 507-522. Springer, 2017.

5. P. Colombo and E. Ferrari. Fine-grained access control within nosql documentoriented datastores. Data Science and Engineering, 1(3):127-138, 092016.

6. R. De Nicola, D. Latella, A. L. Lafuente, M. Loreti, A. Margheri, M. Massink, A. Morichetta, R. Pugliese, F. Tiezzi, and A. Vandin. The SCEL Language: Design, Implementation, Verification, pages 3-71. Springer International Publishing, Cham, 2015.

7. C. Dwork and A. Roth. The algorithmic foundations of differential privacy. Foundations and Trends in Theoretical Computer Science, 9(3-4):211-487, 2013.

8. M. Ercan Nergiz, C. Clifton, and C. Clifton. $\delta$-presence without complete world knowledge m. ercan nergiz, student member, ieee,. 2010.

9. E. Floriano, E. Alchieri, D. F. Aranha, and P. Solis. Providing privacy on the tuple space model. J. Internet Services and Applications, 8(1):19:1-19:16, 2017.

10. R. Hall, A. Rinaldo, and L. Wasserman. Differential privacy for functions and functional data. Journal of Machine Learning Research, 14(1):703-727, 2013.

11. C. Hankin, F. Nielson, and H. R. Nielson. Advice from belnap policies. Computer Security Foundations Symposium, Ieee, pages 234-247, 2009. 
12. B. Kenig and T. Tassa. A practical approximation algorithm for optimal kanonymity. Data Mining and Knowledge Discovery, 25(1):134-168, 2012.

13. N. Li, T. Li, and S. Venkatasubramanian. t-closeness: Privacy beyond k-anonymity and l-diversity. International Conference on Data Engineering (icde), pages 81-+, 2007.

14. A. Lluch-Lafuente, M. Loreti, and U. Montanari. Asynchronous distributed execution of fixpoint-based computational fields. Logical Methods in Computer Science, 13(1), 2017.

15. A. Machanavajjhala, D. Kifer, J. Gehrke, and M. Venkitasubramaniam. l-diversity: Privacy beyond k-anonymity. 2014.

16. A. Margheri, R. Pugliese, and F. Tiezzi. Linguistic abstractions for programming and policing autonomic computing systems. 2013 Ieee 10th International Conference on and 10th International Conference on Autonomic and Trusted Computing (uic/atc) Ubiquitous Intelligence and Computing, pages 404-409, 2013.

17. F. McSherry. Privacy integrated queries: An extensible platform for privacypreserving data analysis. Communications of the Acm, 53(9):89-97, 2010.

18. A. Mehta, E. Elnikety, K. Harvey, D. Garg, and P. Druschel. Qapla: Policy compliance for database-backed systems. In 26th USENIX Security Symposium (USENIX Security 17), pages 1463-1479, Vancouver, BC, 2017. USENIX Association.

19. O. J. of the European Union. Regulation (eu) 2016/679 of the european parliament and of the council of 27 april 2016 on the protection of natural persons with regard to the processing of personal data and on the free movement of such data, and repealing directive 95/46/ec (general data protection regulation). L119:11-88, 05 2016.

20. P. Samarati and L. Sweeney. Protecting privacy when disclosing information: kanonymity and its enforcement through generalization and suppression. Technical report, Harvard Data Privacy Lab, 1998.

21. J. Soria-Comas, J. Domingo-Ferrer, D. Sánchez, and S. Martínez. t-closeness through microaggregation: Strict privacy with enhanced utility preservation. CoRR, abs/1512.02909, 2015.

22. L. Sweeney. k-anonymity: A model for protecting privacy. International Journal of Uncertainty Fuzziness and Knowledge-based Systems, 10(5):557-570, 2002.

23. M. Viroli and F. Damiani. A calculus of self-stabilising computational fields. In eva Kühn and R. Pugliese, editors, Coordination Models and Languages - 16th IFIP WG 6.1 International Conference, COORDINATION 2014, Held as Part of the 9th International Federated Conferences on Distributed Computing Techniques, DisCoTec 2014, Berlin, Germany, June 3-5, 2014, Proceedings, volume 8459 of Lecture Notes in Computer Science, pages 163-178. Springer, 2014. 